\title{
A Reply to Ponte et al (2016) Supply Chain Collaboration: Some Comments on the Nucleolus of the Beer Game
}

\author{
David Mueller \\ Brandenburg University of Technology, Cottbus-Senftenberg (Germany) \\ david.mueller@b-tu.de
}

Received: September 2017

Accepted: March 2018

\begin{abstract}
:
Purpose: The aim of the paper is to pick up the result of a previously published paper in order to deepen the discussion. We analyze the solution against the background of some well-known concepts and we introduce a newer one. In doing so we would like to inspire the further discussion of supply chain collaboration.
\end{abstract}

Design/methodology/approach: Based on game theoretical knowledge we present and compare seven properties of fair profit sharing.

Findings: We show that the nucleolus is a core-solution, which does not fulfil aggregate monotonicity. In contrast the Shapley value is an aggregate monotonic solution but does not belong to the core of every cooperative game. Moreover, we present the Lorenz dominance as an additional fairness criteria.

Originality/value: We discuss the very involved procedure of establishing lexicographic orders of excess vectors for games with many players.

Keywords: beer game, cooperative game theory, profit allocation, Shapley value, nucleolus, core-selection, aggregate monotonicity, Lorenz set

\section{Introduction}

Ponte, Fernández, Rosillo, Parreño and García (2016) suggested in this journal the use of cooperative game theory within supply chain management and demonstrated the application with the famous beer game. Embracing this incorporation of cooperative game theory in principle, some comments and enhancements are necessary.

\section{Aims and Solution Concepts of Cooperative Game Theory}

\subsection{Fundamentals of Game Theory}

Cooperative game theory is based on a range of assumptions. For a detailed discussion we refer to the appropriate literature (Maschler, Solan \& Zamir, 2013, p. 659-662). A cooperative game $\Gamma$ is the pair $(N, v)$, where $N=\{1,2, \ldots$, $n$ \} denotes the set of players. Not only is the amount of all the players $N$ important here, but also all the subsets of $N$. Such a subset $S \subseteq N$ is referred to as coalition $S$, whereby $N$ itself is described as a grand coalition. Each coalition is marked by a value function $v(S)$. The function $v$ assigns a value to each subset $S$, which represents the economic performance of this coalition. 


\subsection{Properties of a Game}

To analyze a solution we have to introduce some classes of games. Cooperation may be successful or not. To concretize the term "success", some desirable properties of games may be defined. One goal is the generation of a result which is not worse than the results of isolated actions. This is referred to as superadditivity. A game $(N, v)$ is superadditive if $v(R \cup S) \geq v(R)+v(S)$ for all $S, R \subseteq N$ with $R \cap S=\varnothing$.

In the following we concentrate on situations in which for at least one coalition yields $v(\mathrm{R} \cup S)>v(\mathrm{R})+v(S)$. In consequence, the grand coalition generates a better result than the sum of all stand-alone coalitions.

A game $(N, v)$ is essential if $v(N)>\sum_{i \in N} v(\{i\})$. By $E^{N}$ we denote the set of all essential games with the set of players $N$. In the following essential games only are analyzed. Superadditivity describes the relationship of coalitions of disjoint elements. A similar effect may be claimed for coalitions of conjoint elements. This is called convexity. A game (N,v) is convex (Maschler et al., 2013, p. 718) if $v(S \cup\{i\})-v(S) \leq v(\mathrm{R} \cup\{i\})-v(\mathrm{R})$ for all $S$ $\subseteq R \subseteq N \backslash\{i\}$. We will denote the set of convex games by $C^{\mathrm{N}}$.

\subsection{Properties of a Fair Solution}

Looking at a game, the question arises of how to share the jointly generated result between the partners, which is equivalent to an allocation of the result. A function $f(v)$ which assigns to a game $(N, v)$ a, possible empty, subset $f(v)$ of $\mathbb{R}^{\mathrm{N}}$ is called a solution concept. The function $f$ distributes $v(N)$ and generates a payoff vector $x=\left(x_{1}, x_{2}, x_{3}, \ldots x_{n}\right)$ with $\mathrm{x} \in \mathbb{R}^{\mathrm{N}}$. Such a function is referred to as allocation scheme.

Definition 1: A solution $f$ is a single-valued solution if $|f(v)|=1$ for every $v$. In this case, $f(v)$ is represented by an element of $\mathbb{R}^{\mathrm{N}}$, i.e. $f(v)=x$.

With the allocation of the jointly generated result, the problem of fairness arises. Several properties of a fair solution have been identified in cooperative game theory in the last decades. The most crucial properties are (González-Díaz, García-Jurado \& Fiestras-Janeiro, 2010, p. 226; Calleja, Rafels \& Tijs, 2012; Mueller, 2018, p. 406408):

- Efficiency: A single-valued solution $f$ is efficient if $\sum_{i \in N} f_{i}(v)=v(N)$.

- Individual rationality: A single-valued solution $f$ is individual rational if $f_{i}(v) \geq v(\{i\}) \forall i \in N$.

- Equal-treatment-property: A single-valued solution $f$ satisfies equal-treatment property if for the players $i$ and $j$, for which holds: $v(S \cup\{i\})=v(S \cup\{j\}) \forall S \subseteq N$ with $i, j \notin S$ yields $f_{i}(v)=f_{j}(v)$.

- Dummy-player-property: A player $i$ is called a dummy player if $v(S \cup\{i\})=v(S)+v(\{i\})$ for all $S \subseteq N$ with $i \notin S$. A single-valued solution $f$ satisfies the dummy-player-property if for a dummy-player $i$ yields: $f_{i}(v)=v(\{i\})$.

- Additivity: A single-valued solution $f$ satisfies additivity if for any two games $v, w$ follows $(v+w)=f(v)+f(w)$.

- Aggregate monotonicity: A single-valued solution $f$ satisfies aggregate monotonicity if for all games $v, w$ with $v(N)>w(N)$ and $v(S)=w(S)$ for all $S \subsetneq N$ follows: $f_{i}(v) \geq f_{i}(w) \forall i \in N$.

There are several other properties (Arin \& Katsev, 2018, p. 305) which are not of interest for further discussion. The first two properties are summerized by defining an imputation.

Definition 2: The set of imputations $I(v)$ of a game $N(v)$ is defined by

$$
I(v)=\left\{x \in \mathbb{R}^{\mathrm{N}} \mid \sum_{i \in N} x_{i}=v(N) \text { and } x_{i} \geq v(\{i\}) \forall i \in N\right\} \text {. }
$$

Only those imputations are of interest that are not dominated by another imputation. The set of non-dominated imputations forms the core. 
Definition 3: The Core $(v)$ of a game $N(v)$ is defined by

$$
\operatorname{Core}(v)=\left\{x \in \mathrm{I}(v) \mid \sum_{i \in S} x_{i} \geq v(S) \forall S \subseteq N\right\}
$$

The core of a game contains all solutions which are justified as fair and, therefore, are stable. It may be small, very large, or empty. Without defining the property of balancedness in detail we point out, that the core of a balanced game is never empty (Mueller, 2018, p. 405).

By $B^{N}$ we denote the set of all balanced games with player set $N$. With this class at hand we introduce the core-selection-property. A solution satisfies core selection if it selects a core element for any game with a non-empty core.

Core selection: A single-valued solution $f$ satisfies core selection if $f(v) \in \operatorname{Core}(v)$ for all $v \in B^{N}$.

\subsection{Characterization of the Shapley-Value and the Nucleolus}

In order to determine a fair share for player $i$, the following thought is worth noting: each player receives a part depending on that player's contributions to the theoretically possible, thus imaginable, coalitions. The contribution of the player consists in the increase in value caused by that player's participation in the coalition. The question that has to be answered is which value the coalition has with player $i$ and which it would have without player $i$. This difference is called the marginal contribution.

Definition 4: The marginal contribution $m c_{i}$ of player $i$ to a coalition $S$ is defined by: $m c_{i}=v(S \cup\{i\})-v(S)$.

Assuming all orders of forming a coalition to have the same probability results in the weighted average of the marginal contributions of a player, which is commonly described as the Shapley value (Shapley, 1953, p. 311).

Definition 5: The Shapley value of a player $\varphi_{i}$ in a game $(N, v)$ is defined by

$$
\varphi_{i}=\sum_{S \subseteq N \backslash\{i\}} \frac{|S| !(n-1-|S|) !}{n !}[v(S \cup\{i\})-v(S)] .
$$

The Shapley-value (Mueller, 2018, p. 412):

- $\quad$ satisfies efficiency, dummy-player-property, equal-treatment-property, additivity and aggregate monotonicity for all cooperative games,

- $\quad$ is individual rational for each $v \in E^{N}$,

- but fulfils core-selection only for each $v \in C^{N}$.

The nucleolus was introduced by Schmeidler (1969) and searches for a fair distribution by minimizing the maximal dissatisfaction of every player. To achieve this, the dissatisfaction of a coalition with a concrete payoff vector is named in this connection as excess. It is necessary to calculate how unhappy a coalition would be with a payoff vector.

Definition 6: The excess (unhappiness) $\operatorname{ex}(S, x)$ of a coalition $S$ with a payoff vector $x$ is derived by $\operatorname{ex}(S, x)=v(S)-\sum_{i \in S} x_{i}$

To derive the nucleolus, the payoff vectors with the highest unhappiness for every player are sought in the next step. To do so, these excess values are sorted in non-increasingly order (González-Díaz et al., 2010, p. 233; Mueller, 2016, p. 205). The excess of a coalition $S_{i}$ with respect to a payoff vector $x$ is denoted by $e x\left(S_{i}, x\right)=\theta_{i}(x)$.

Definition 7: The vector of non-increasingly ordered excess values $\Theta$ is defined by $\Theta(x)=\theta_{1}(x) ; \theta_{2}(x) ; \theta_{3}(x) ; \ldots$; $\theta_{2}^{\mathrm{n}}(x)$ with $\theta_{i}(x) \geq \theta_{j}(x)$ for $1 \leq i \leq j \leq 2^{n}$. 
To compare two payoffs, their vectors of non-increasingly ordered excess values are compared based on the lexicographic order. The vector which is lexicographically smaller than the other one is chosen as this vector offers the minimum of the maximal dissatisfaction for all players resulting from the two payoffs.

Definition 8: If two imputations $x$ and $y$ are compared, then $x$ is considered to be lexicographically smaller $\left(\prec_{\text {LEX }}\right)$ than $y$ if there exists an index $m$, with which results $\theta_{k}(x)=\theta_{k}(y) \forall 1 \leq k \leq m$ and $\theta_{m}(x)<\theta_{m}(y)$.

With these explanations, the nucleolus of a game can be defined as follows (González-Díaz et al., 2010, p. 232):

Definition 9: In a game $(N, v)$ with $I(v) \neq \varnothing$ the nucleolus $n u c(v)$ is defined by: $n u c(v)=\left\{x \in I(v) \mid \Theta(x) \prec_{\text {LEX }} \Theta(y)\right.$ $\forall y \in I(v)\}$.

We summarize that the nucleolus (Mueller, 2018, p. 414):

- satisfies efficiency, individual rationality, dummy-player-property, equal-treatment-property and core selection for each $v \in E^{N}$,

- does not fulfil additivity for each $v \in E^{N}$,

- $\quad$ is neither for each $v \in E^{N}$ nor for each $v \in C^{|N| \geq 4}$ aggregate monotonic.

\subsection{Analyzing the Beer Game}

Table 1 contains the characteristic function of the beer game.

\begin{tabular}{|c|c|c|c|c|c|c|c|}
\hline$S$ & $v(S)$ & $S$ & $v(S)$ & $S$ & $v(S)$ & $S$ & $v(S)$ \\
\hline$\{\varnothing\}$ & 0 & $\{4\}$ & 400 & $\{2,3\}$ & 550 & $\{1,2,4\}$ & 850 \\
\hline$\{1\}$ & 100 & $\{1,2\}$ & 400 & $\{2,4\}$ & 650 & $\{1,3,4\}$ & 1,250 \\
\hline$\{2\}$ & 200 & $\{1,3\}$ & 450 & $\{3,4\}$ & 750 & $\{2,3,4\}$ & 1,050 \\
\hline$\{3\}$ & 300 & $\{1,4\}$ & 600 & $\{1,2,3\}$ & 800 & $\{1,2,3,4\}$ & 1,500 \\
\hline
\end{tabular}

Table 1. Characteristic function of the beer game. Ponte et al. (2016, p. 1027).

Using the notation $\{1\}=\mathrm{S},\{1,4\}=\mathrm{R}$, and $\{\mathrm{i}\}=2$ shows that the game is not convex as we get: $400-100 \$ 850-600$. To guide the following procedure, we have to check if the game has a non-empty core at all. Establishing a system of inequalities based on the characteristic function shows that this question can be answered in the affirmative. So, the core is not empty and there exists a fair solution.

Computing the Shapley values for the players leads to the following results: $\varphi_{1}=262.50, \varphi_{2}=254 \frac{1}{6}, \varphi_{3}=445 \frac{5}{6}$ and $\varphi_{4}=537,50$. As pointed out, the non-convexity of the game may cause that the Shapley-value does not belong to the core. Analyzing coalition $\{1,3,4\}$ shows that these players get a value of $1,245 \frac{\mathbf{5}}{\mathbf{6}}$. This is less than the value which they generate $(1,250)$. That's why the Shapley value is not a core-allocation and we have to compute the nucleolus.

Concerning the calculational effort of the nucleolus, the very involved procedure of establishing lexicographic orders of excess vectors for games with many players must be mentioned. There are some mistakes in computing the nucleolus caused by overlooking the possibility that a linear program can have multiple solutions (Guajardo \& Jørnsten, 2015). Nevertheless, the nucleolus has been correctly computed in several publications (e. g. Fromen, 1997; Hallefjord, Helming \& Jørnsten, 1995; Kimms \& Çetiner, 2012).

The solution of the beer-game is $x=(225,225,410.5,639.5)$ (Ponte et al., 2016, p. 1029). Checking Definition 3 indicates that this solution belongs to the non-empty core. That means it is a stable and fair solution. Unfortunately, this result is not the nucleolus. To prove that we compute the resulting vector of non-increasingly ordered excess values $\Theta(x)$. We get: 


$$
\Theta(x)=(0,0,-25,-25,-50,-60.5,-85.5,-110.5,-125,-185.5,-214.5,-225,-239.5,239.5,-264.5,-300) .
$$

We may reduce the maximal dissatisfaction by choosing the imputation $y=(250,225,410.50,614.5)$. This leads to the vector of non-increasingly ordered excess values $\Theta(y)$, with:

$$
\Theta(y)=(0,0,-25,-25,-75,-85.5,-85.5,-110,5 .-150,-189.5,-200,-210.5,-214.5,-239.5,-264.5,-275) .
$$

Comparing these vectors, we can conclude that $\Theta(y) \prec_{\mathrm{LEX}} \Theta(x)$, what indicates that $x$ is not the nucleolus. Continuing the procedure, we get the nucleolus with $\operatorname{nuc}(v)=\left(291 \frac{2}{3}, 225,441 \frac{2}{3}, 541 \frac{2}{3}\right)$. This generates the following vector of non-increasingly ordered excess values:

$$
\Theta(n u c)=\left(0,0,-25,-25,-116 \frac{2}{3},-116 \frac{2}{3},-116 \frac{2}{3},-141 \frac{2}{3},-141 \frac{2}{3},-158 \frac{1}{3},-158 \frac{1}{3},-191 \frac{2}{3},-208 \frac{1}{3},-233 \frac{1}{3},-233 \frac{1}{3},-283 \frac{1}{3}\right) .
$$

This result differs significantly from the original value. But we have pointed out that both values belong to the nonempty core. The problem is that the core of the discussed beer game is large and contains a lot of possible allocations. So the question rises for a justification of an allocation which is wider than the core argumentation. Besides the presented nucleolus we want to present another possible argument - the Lorenz set.

\subsection{Lorenz Dominance, the Lorenz Set, and the Lorenz Solution}

Lorenz dominance was established to mirror the concentration of wealth in a quantitative way. Starting point of the argumentation is a society of $n$ individuals in which the total income of $I$ is distributed by the allocation $x$ (Brânzei, Dimitrov \& Tijs, 2008, p. 37). The vector $\hat{x}$ results from rearranging $x$ according to $\hat{x}=\left(\hat{x}_{1} \leq \hat{x}_{2} \leq \cdots \leq \hat{x}_{n}\right)$.

The vector $\hat{x}$ Lorenz dominates the vector $\hat{y}$ for any $x, y \in \mathbb{R}_{+}^{n}$ with $\sum_{i=1}^{n} x_{i}=\sum_{i=1}^{n} y_{i}=I$

if $\sum_{i=1}^{p} \hat{x}_{i} \geq \sum_{i=1}^{p} \hat{y}_{i}$ for all $p \in\{1, \ldots, n-1\}$ with at least one strict inequality. In this case we denote $x \succ_{\text {LOR }} y$.

Lorenz dominance implies an allocation with less inequality. Some concepts of egalitarian solution have been developed based on Lorenz dominance (cf. Arin, Kuipers \& Vermeulen, 2008: p. 569-571). As the beer game is not convex we present a newer solution concept - the Lorenz set (Hougaard, Peleg \& Thorlund-Petersen, 2001; Hougaard \& Smilgins, 2016).

Definition 10: The Lorenz set $L(v)$ of a game is defined by:

$$
L(v)=\left\{x \in \operatorname{Core}(v) \mid \nexists \mathrm{y} \in \operatorname{Core}(v): y \succ_{\mathrm{LOR}} x\right\} .
$$

The Lorenz set consists of Lorenz undominated solutions, which belong to the core. We can state that $L(v) \subseteq \operatorname{Core}(v)$ and that $L(v) \neq \varnothing$ if $\operatorname{Core}(v) \neq \varnothing$ (Hougaard \& Smilgins, 2016, p. 153).

The Lorenz set coincides with equal-distribution-solution if this solution belongs to the core. If the equal distribution does not belong to the non-empty core, then there exists a unique allocation $x \in \operatorname{Core}(v)$, which minimizes the Euclidian distance from the equal distribution to the core and $x \in L(v)$. This is the Lorenz-solution based on least squares solution (Arin et al., 2008, p. 569). We define the Euclidean length $\|x\|$ for an allocation $\mathrm{x}$ with: $\|x\|=\sqrt{\sum_{i \in N} x_{i}^{2}}$.

Definition 11: The Lorenz solution $L S(v)$ of a balanced game is the allocation $x \in \operatorname{Core}(v)$ for which $\|x\| \leq\|y\|$ for all $y \in \operatorname{Core}(v)$. 
To analyze the nucleolus and the solution of Ponte et al. we will rearrange the values in non-decreasing order what leads to $\hat{x}_{1}=(225,225,410.5,639.5)$ for the solution of Ponte et al. and $\hat{x}_{2}=\left(225,291 \frac{2}{3}, 441 \frac{2}{3}, 541 \frac{2}{3}\right)$ for the nucleolus. It becomes apparent that the nucleolus Lorenz dominates the other solution. But the nucleolus is not a member of the Lorenz set. If we have a look at the imputation $x_{3}=\left(416_{3}^{\frac{2}{3}}, 250,416_{3}^{\frac{2}{3}}, 416^{\frac{2}{3}}\right)$, we get the ordered vector $\hat{x}_{3}=\left(250,416_{3}^{\frac{2}{3}}, 416_{3}^{\frac{2}{2}}, 416_{3}^{-}\right)$. This vector Lorenz dominates all the other vectors and minimizes the Euclidean distance to the equal distribution.

\section{Summary}

Incorporating game theoretic solution concepts into supply chain collaboration is a welcome broadening for the management of this process. Interpreting such situations as a cooperative game may lead to some useful insights. Beside the well-established concepts we have introduced a newer concept of fairness. The original results were reflected against the background of the new solution. We have shown that the nucleolus is a useful concept for balanced games which are not convex. The Lorenz solution enriches the discussion by introducing the Lorenzean understanding of fairness. In doing so we would like to inspire the further discussion of supply chain collaboration.

\section{Declaration of Conflicting Interests}

The author declared no potential conflicts of interest with respect to the research, authorship, and/or publication of this article.

\section{Funding}

The author received no financial support for the research, authorship, and/or publication of this article.

\section{References}

Arin, J., \& Katsev, I. (2018). The SD-prenucleolus for TU-Games: coalitional monotonicity and core stability. In Mueller, D., \& Trost, R. (Eds.), Game theory in management accounting - implementing incentives and fairness (301-321). Berlin et al.: Springer.

Arin, J., Kuipers, J., \& Vermeulen, D. (2008). An axiomatic approach to egalitarianism in TU-games. International Journal of Game Theory, 37(4), 565-580. https://doi.org/10.1007/s00182-008-0133-6

Brânzei, R., Dimitrov, D., \& Tijs, S.H. (2008). Models in Cooperative Game Theory (2nd ed.). Berlin.

Calleja, P., Rafels, C., \& Tijs, S.H. (2012). Aggregate monotonic stable single-valued solutions for cooperative games. International Journal of Game Theory, 41(4), 899-913. https://doi.org/10.1007/s00182-012-0355-5

Fromen, B. (1997). Reducing the number of linear programs needed for solving the nucleolus problem of $n$-person game theory. European Journal of Operational Research, 98(3), 626-636. https://doi.org/10.1016/0377-2217(95)00341-X

González-Díaz, J., García-Jurado, I. \& Fiestras-Janeiro, M.G. (2010). An introductory course on mathematical game theory. Providence: American Mathematical Society. https://doi.org/10.1090/gsm/115

Guajardo, M., \& Jørnsten, K. (2015). Common mistakes in computing the nucleolus. European Journal of Operational Research, 241(3), 931-935. https://doi.org/10.1016/j.ejor.2014.10.037

Hallefjord, A., Helming, R., \& Jørnsten, K. (1995). Computing the nucleolus when the characteristic function is given implicitly: a constraint generation approach. International Journal of Game Theory, 24(4), 357-372. https://doi.org/10.1007/BF01243038

Hougaard, J.L., Peleg, B., \& Thorlund-Petersen, L. (2001). On the set of Lorenz-maximal imputations in the core of a balanced game. International Journal of Game Theory, 30(2), 147-165. https://doi.org/10.1007/s001820100070

Hougaard, J.L., \& Smilgins, A. (2016). Risk capital allocation with autonomous subunits: The Lorenz set. Insurance: Mathematics and Economics, 67(3), 151-157. https://doi.org/10.1016/j.insmatheco.2015.12.002 
Kimms, A., \& Çetiner, D. (2012). Approximate nucleolus-based revenue sharing in airline alliances. European Journal of Operational Research, 220(2), 510-521. https://doi.org/10.1016/j.ejor.2012.01.057

Maschler, M., Solan, E., \& Zamir, S. (2013). Game theory. Cambridge: Cambridge University Press. https://doi.org/10.1017/CBO9780511794216

Mueller, D. (2016). What's in it for me? - An analysis of environmental investments through a game theoretic lens. International Journal of Innovation and Sustainable Development, 10(2), 198-218.

https://doi.org/10.1504/IJISD.2016.075551

Mueller, D. (2018). The usability and suitability of allocation schemes for corporate cost accounting. In Mueller, D., \& Trost, R. (Eds.), Game theory in management accounting - implementing incentives and fairness (401-427) Berlin et al.: Springer. https://doi.org/10.1007/978-3-319-61603-2_19

Ponte, B., Fernández, I., Rosillo, R., Parreño, J., \& García, N. (2016). Supply chain collaboration: a game-theoretic approach to profit allocation. Journal of Industrial Engineering and Management, 9(5), 1020-1034. https://doi.org/10.3926/jiem.2084

Schmeidler, D. (1969). The nucleolus of a characteristic function game. Journal of Applied Mathematics, 17(6), 1163-1170. https://doi.org/10.1137/0117107

Shapley, L.S. (1953). A value for n-person games. In Kuhn, H.W., \& Tucker, A.W. (Eds.), Contributions to the theory of games (II) (307-317). Princeton: Princeton Univ. Press. https://doi.org/10.1515/9781400881970-018

Journal of Industrial Engineering and Management, 2018 (www.jiem.org)

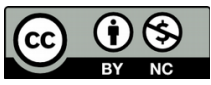

Article's contents are provided on an Attribution-Non Commercial 4.0 Creative commons International License. Readers are allowed to copy, distribute and communicate article's contents, provided the author's and Journal of Industrial Engineering and Management's names are included. It must not be used for commercial purposes. To see the complete license contents, please visit https://creativecommons.org/licenses/by-nc/4.0/. 